hospitalisation for coronary heart disease (CHD). Follow-up was subsequently performed at least 12 months after the interview to record new CVD events. The primary outcome was a composite of fatal and non-fatal CVD. Multivariable-adjusted Cox regression analysis was performed.

Results Of the 8251 participants who attended the interview, 2126 (25.8\%) were female. The majority (7060, 86\%) were Caucasian. In total, $1303(16.7 \%)$ had CKD. The prevalence of CVD risk factors are outlined in table 1. At follow-up 7509 (98.5\%) were alive. Of those that died, 37 (51.39\%) deaths were due to CHD (figure 1). Regarding non-fatal events, $135(1.8 \%)$ were hospitalised for myocardial infarction (MI) during follow-up and $84(1.1 \%)$ were hospitalised for a stroke/TIA. Among those with recurrent CVD events (i) mean eGFR (95\% CI) was $14.9 \mathrm{ml} / \mathrm{min} / 1.73 \mathrm{~m}^{2}$ lower in persons suffering fatal CVD vs. others $(6.5,23.4) \mathrm{p}=0.001$ (ii) eGFR was $7.23 \mathrm{ml} / \mathrm{min} / 1.73 \mathrm{~m}^{2}$ lower in stroke than no stroke (2.16, 12.31) $\mathrm{p}=0.006$ (iii) eGFR was $1.56 \mathrm{ml} / \mathrm{min} / 1.73 \mathrm{~m}^{2}$ lower in MI than no MI $(-3.03,6.14) \mathrm{p}=0.503$. (Table 2, figure 2) Adjusted hazard ratio (95\% CI) for CV events in those with CKD versus those without: (i) CVD Death 1.305 $(0.519,3.279) \mathrm{p}=0.572$ (ii) MI $1.228(0.753,2.000) \mathrm{p}=$ 0.410 (iii) Stroke $1.563(0.864,2.828) \mathrm{p}=0.14$.

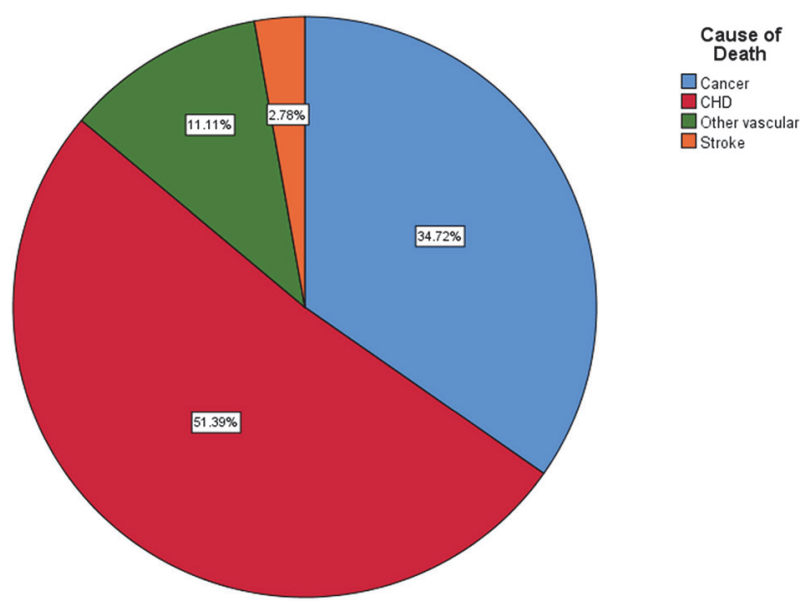

Abstract 27 Figure 1 Known cause of death $(n=72)$. CHD, coronary heart disease

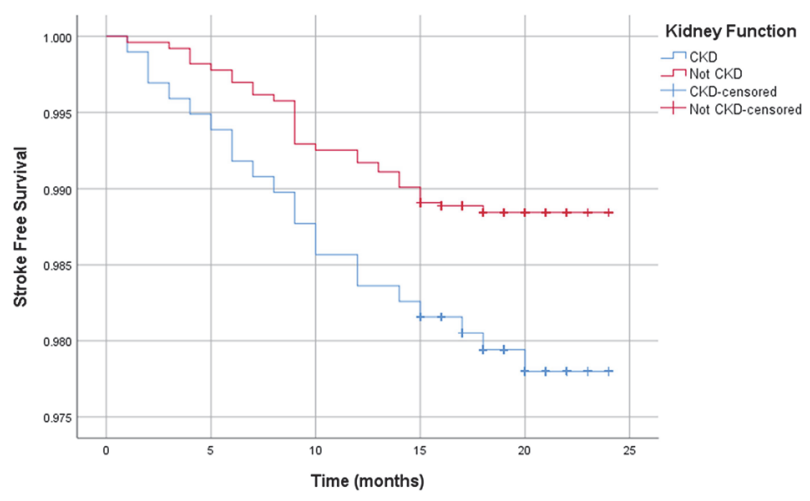

Abstract 27 Figure 2 Kaplan meier analysis

There is a statistically significant association between CKD and stroke (Log Rank test $p$ value 0.012), indicating an increased likelihood of having a stroke over follow-up in months in those with CKD versus those without
Conclusion A sixth of the European CHD population has CKD. Participants who had a stroke or died from CVD over follow-up had a statistically significantly lower baseline eGFR than those who did not. The HR suggest an increased risk for CV events in CKD but this was not statistically significant. Further studies with a longer follow-up period are needed in this area.

\section{PERCUTANEOUS MITRAL VALVE REPAIR WITH MITRACLIP DEVICE IN HEMODYNAMICALLY UNSTABLE PATIENTS: A SYSTEMATIC REVIEW}

A McInerney, E Martinez-Gomez, G Triado-Contre, L Nombela-Franco. Hospital Clinico San Carlos, Madrid, Spain

\subsection{6/heartjnl-2020-ICS.28}

Background Percutaneous mitral valve edge-to-edge repair (PMVr) with the MitraClip device has been reserved as an alternative treatment for chronic mitral regurgitation (MR) in high surgical risk patients. Acute significant $M R$ has been reported to complicate $12 \%$ of myocardial infarctions and is present in 5 to $12 \%$ of patients in cardiogenic shock. The use of MitraClip in hemodynamically unstable patients, who are of high surgical risk, is therefore increasing. However, there is a paucity of specific outcome data in this important clinical setting as these patients are generally not included in clinical trials.

Objectives The aim of the present study was to perform a systematic review of published data, and provide further insight into the baseline characteristics, management and clinical outcomes of unstable patients undergoing PMVr with MitraClip.

Methods We conducted a systematic review of the published data on MitraClip from its first use in 2003 to February 2020. Pubmed search terms included 'Acute' and 'MitraClip', and later 'MitraClip' and 'cardiogenic shock'. Studies referring to critically ill patients in cardiogenic shock or acute pulmonary edema were included. Studies referring to stable patients were excluded.

Results A total of 27 publications including 155 patients with significant MR (grade 4 in 91\%) were included. Mean age was $70 \pm 12$ years with median Euroscore II and STS of 15.7 (IQR 10.5-27.6) and 18.1 (IQR 7.6-23.9), respectively. Clinical presentation was with cardiogenic shock in $77.5 \%$ of patients. The most frequent etiology of MR was ischemia (77\%). Median time between hospital admission and MitraClip implantation was 22 (IQR 8-45) days. Hemodynamic support was required in the majority of the patients $(87.1 \%)$, with vasoactive drugs and ventricular support in 101 patients (65.2\%) and 67 patients (43.2\%), respectively. MR was quantified as severe (grade 4) in $91.3 \%$ of the patients, with $15.9 \%$ related to a papillary muscle rupture in the context of acute STEMI. Left ventricular ejection fraction (LVEF) was moderate-to-severely depressed (34.2 $\pm 15.6 \%)$. Device success was achieved in 147 (95\%) patients with a significant reduction in $\mathrm{MR}$ (grade $\leq 2$ in $94 \%, \mathrm{p}<0.001$ ). The weaning time from the procedure, to discontinuation of circulatory or respiratory support, was 2.5 (IQR 1.0-6.8) days, with an inhospital mortality and non-fatal complication rate of $9 \%$ and $5.2 \%$, respectively. At 6-month follow-up, mortality rate was $21.5 \%$, with persistent reduction in MR severity for survivors (grade $\leq 2$ in $83 \%$ ) and one case of mitral valve reintervention. 
Conclusions Percutaneous mitral valve repair with MitraClip device is a safe and viable management option in high-risk patients with cardiogenic shock or refractory pulmonary edema and concomitant moderate-severe MR. Prospective trials are required to confirm these findings, and definitively determine the value of MitraClip in hemodynamically unstable patients.

\section{SAFETY AND EFFICACY OF TRANSCATHETER AORTIC VALVE IMPLANTATION IN NONAGENARIANS WITH SEVERE SYMPTOMATIC AORTIC STENOSIS}

PF Brennan, B Cox, A Santos, F Corvan, O Nzewi, N Johnston, A McNeice, R Jenanathan, C Owens, MS Spence, G Manoharan. Royal Victoria Hospital, Belfast, UK

\subsection{6/heartjnl-2020-ICS.29}

Background Transcatheter aortic valve implantation (TAVI) is the standard of treatment for high/prohibitive surgical risk patients with symptomatic severe aortic stenosis. Studies reported over the last 5 years have found TAVI to be noninferior to surgery with respect to intermediate and lower risk patients. In the United Kingdom the number of people living beyond 90 years age continues to rise and this had led to an increasing number of nonagenarians undergoing TAVI for symptomatic aortic stenosis. Outcomes for this group patients are scarcely reported.

Purpose We aimed to report outcomes for all nonagenarians undergoing TAVI in Northern Ireland (NI).

Methods Baseline characteristics, procedural parameters and outcomes were collected using the NICOR database, NI Electronic Care Record and our in-hospital database. We compared all 73 nonagenarians with the remaining 1048 patients that underwent TAVI in NI.

Results Nonagenarians (mean age $=91.6$ years; female sex $55 \%)$ had a lower mean EuroSCORE2 $(7.2 \%$ vs $8.5 \%$, $\mathrm{p}=0.03$ ) than the younger group (mean age $=81$ years; female sex $53 \%)$. COPD $(4.1 \%$ vs. $20.3 \%, \mathrm{p}<0.005)$, diabetes mellitus $(11 \%$ vs. $22.3 \%$, p 0.02$)$, prior cardiac surgery $(6.8 \%$ vs. $19.8 \%, \mathrm{p} 0.01)$ were all significantly lower in the nonagenarian patients. Nonagenarians had significantly more progressive renal dysfunction (mean creatinine clearance $36.4 \mathrm{ml} / \mathrm{min}$ vs. $48.3 \mathrm{ml} / \mathrm{min}, \mathrm{p}<0.005)$.

Left ventricular dysfunction $(\mathrm{LVEF}<50 \%)$ was less prevalent in the nonagenarians ( $14 \%$ vs $28 \%$, p 0.01). TAVI was performed under local anaesthetic (LA) in $97 \%$ of nonagenarians, compared to $95.5 \%$ younger patients, for which 93\% vascular access was percutaneous femoral (pTF) (nonnonagenarians LA 98\%, pTF 92.2\%). Surgical femoral and axillary/subclavian made up the remainder of the access for nonagenarians.

Procedural complications for nonagenarians vs the remaining group included stroke $(4.3 \%$ vs $2.82 \%$, p 0.22), tamponade $(1.4 \%$ vs $1 \%, \mathrm{p} 0.61)$ and major vascular injury $(1.4 \%$ vs $3.4 \%$, p 0.62 ). $30 \mathrm{~d}$ new pacemaker implantation was similar between the nonagenarian and younger patients (12 vs. $13 \%$, p 0.78). Peri-procedural mortality trended higher in nonagenarians at $4 \%$ vs $2 \%$, p 0.20 but, overall, $30 \mathrm{~d}(4 \%$ vs. $3 \%, \mathrm{p} 0.47)$ and $1 \mathrm{y}(16 \%$ vs. $10 \%, \mathrm{p} 0.10)$ mortality did not significantly differ when compared with the younger patient group. The median survival estimate (figure 1) for nonagenarians was 4.9y (IQR 2.3-7.4y) compared to 6.1y (IQR 3.1$9.1 y$ ) for the younger patient group ( $p$ 0.10). Readmission for

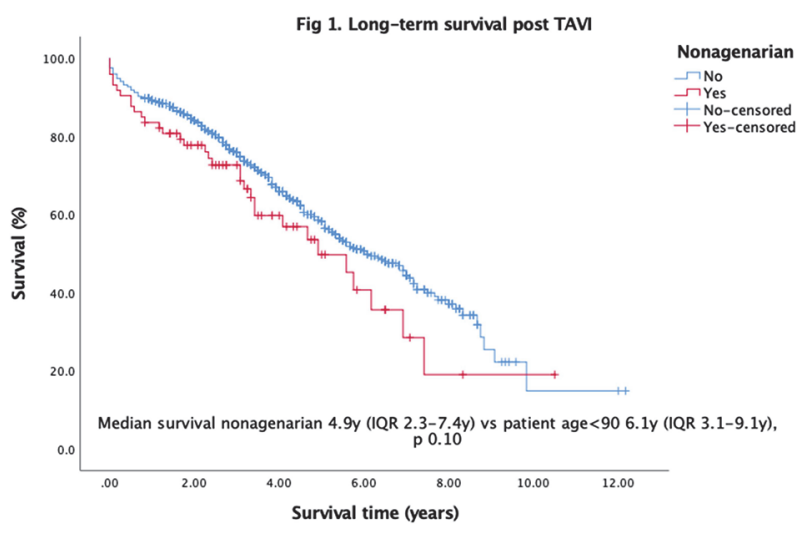

Abstract 29 Figure 1 The median survival estimate

heart failure within 1-year was non-significantly lower for nonagenarians (6\% vs $10 \%$, p 0.23).

Conclusions In selected nonagenarians we severe symptomatic aortic stenosis, TAVI is both safe and efficacious achieving acceptable short- and long-term outcomes that are comparable with younger patients undergoing TAVI.

\section{SYNTAX SCORE- A SIMPLE WAY TO STRATIFY COMPLEX CORONARY DISEASE?}

J Mailey, C Murphy. Royal Victoria Hospital, Belfast, UK

\subsection{6/heartjnl-2020-ICS.30}

Background The Syntax Score (SS) is an angiographic risk stratification tool to grade coronary disease complexity and aid revascularisation strategy decision making. Calculated scores are divided into tertiles $(0-22,23-32,>32)$ and guidelines advocate that decisions should be based on this tertile (1). Syntax Score calculation is dependent on the subjective interpretation of coronary angiograms by Interventional Cardiologists (ICs) and is therefore prone to variability. Our institution consists of 13 ICs performing on average >5000 coronary procedures per year. We sought to compare syntax scoring amongst ICs.

Methods 10 patients with multi-vessel coronary artery disease who had previously been discussed by a Multi-Disciplinary team (MDT) were retrospectively identified. Eight ICs were asked to interpret the coronary angiograms and calculate a SS for each patient. Scoring was performed independently, with no knowledge of the score that had been used at the MDT discussion. Interobserver variability between raters for the raw syntax score was assessed using intraclass correlation coefficient. Interobserver variability between raters with respect to tertile was assessed using Fleiss kappa.

Results All 8 ICs completed scores for each patient. Mean calculated SS was $30.2( \pm 6.7 \mathrm{SD}) .21 .3 \%$ of scores were $1 \mathrm{st}$ tertile (0-22), $45.0 \%$ were 2 nd tertile (23-32) and $33.7 \%$ were 3rd tertile $(>33)$. Mean range of calculated SS between raters was 18.5 (range 9.5 - 29). 40.0\% of scores were divided across all 3 tertiles with the remaining $60.0 \%$ divided across 2 tertiles. Intraclass correlation coefficient of raw SS was 0.55 suggesting only fair agreement. The interobserver strength of agreement with regard to tertiles was also only fair (Fleiss Kappa $=0.25$; 95\% CI [0.07, 0.43]). The interobserver strength of agreement improved to moderate when comparing tertile 1 vs tertile 2 or 3 together (Fleiss 